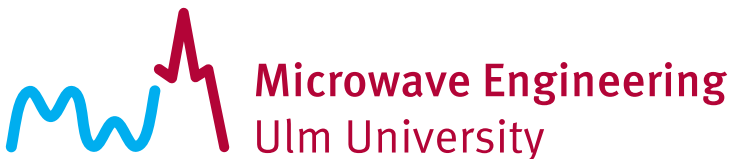

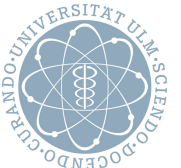

\section{Implementation of a SAR Demonstrator for Automotive Imaging}

Hasan Iqbal, Markus Schartel, Fabian Roos, Jürgen Urban, and Christian Waldschmidt

(C) 2018 IEEE. Personal use of this material is permitted. Permission from IEEE must be obtained for all other uses, in any current or future media, including reprinting/republishing this material for advertising or promotional purposes, creating new collective works, for resale or redistribution to servers or lists, or reuse of any copyrighted component of this work in other works. 


\title{
Implementation of a SAR Demonstrator for Automotive Imaging
}

\author{
Hasan Iqbal, Markus Schartel, Fabian Roos, Jürgen Urban and Christian Waldschmidt \\ Institute of Microwave Engineering, Ulm University, 89081 Ulm, Germany \\ Email: hasan.iqbal@uni-ulm.de
}

\begin{abstract}
To fulfil the need for finer resolution automotive radar imaging, in this paper, a SAR demonstrator and processing algorithm at $77 \mathrm{GHz}$ are shown which represent a realistic low cost implementation for automotive applications.

In the measurement setup section, the demonstrator is described along with how the strict requirements of synchronous radar measurements and trajectory information are fulfiled. A processing algorithm is also presented that is modelled for automotive applications, such that the measurements are processed in blocks, which represent sub-apertures, as they become available from the radar. This results in a faster processing time. Additionally the effect of the missing signals in azimuth, which occur because of hardware restrictions, is clarified and minimised using compressed sensing by recovering the missing signals.

To verify the results delivered from the developed algorithm, the processed images are compared with those obtained from the time domain backprojection algorithm and the results were found to be in good agreement.
\end{abstract}

\section{INTRODUCTION}

There has been a significant amount of research done in the implementation of synthetic aperture radar (SAR) for automotive applications due to its ability to deliver high resolution radar images. With SAR, one is able to achieve radar images with a very fine resolution of objects which is vital for efficient functioning of driver assistance and safety systems. Whereas a lidar sensor for example is severely affected by fog and rain, a radar sensor faces only a minor blemish in performance.

The authors of [1] and [2] have published results using SAR demonstrators. However both of them utilised an elaborate measurement setup with multiple sensors for trajectory tracking which did not yield a realistic real time capable implementation. In [3], automotive SAR is presented employing the range migration algorithm (RMA), however this implementation too is not real-time capable. None of these papers mention whether there were any missing signals in azimuth (due to implementation or hardware requirements) and if so whether they were recovered. Thus these implementations do not represent a realistic real time solution for automotive applications. A real time implementation of SAR using the time domain backprojection algorithm has also been reported [4]. This algorithm though is considerably more expensive with respect to the processing time when compared to RMA [5], which has been used in this work, for example.

It was previously demonstrated that the SAR algorithms, although sensitive to motion errors, can be made robust against inaccuracies of inertial measurement units (IMUs) or acceleration sensors which are typically used in cars, for example by reducing the length of the 'synthetic aperture' [6]. Building upon that, the goal of the research for this work was to present a low cost realistic SAR implementation using minimal hardware as a proof of concept. The signal processing algorithm has been developed so that it reflects the realistic real-time setup if it should be implemented in a car. The presented algorithm is faster and therefore well suited for a real-time implementation. Also the gaps between signals in azimuth (due to hardware restrictions, discussed in Section II-B) have been filled by reconstructing the missing signals with the help of compressed sensing [7]. Additionally the processed images are verified by comparison against the same measurements processed through the backprojection algorithm [8].

\section{Measurement Setup and Algorithm IMPLEMENTATION}

The $77 \mathrm{GHz}$ radar sensor used to carry out these measurements [6] was fixed on a trolley such that the radar boresight direction is perpendicular to the trajectory of the trolley. The height of the sensor above ground was $40 \mathrm{~cm}$, which represents the height of a typical automotive radar located in the bumper of a car. The bandwidth used for the measurements was $4 \mathrm{GHz}$. The trajectory information was captured with the help of a rotary encoder which was attached to the wheel of the trolley. This rotary encoder had a resolution of $0.3 \mathrm{~mm}$ which is more than enough for this application. No other means of capturing the trajectory information were utilised.

The rotary encoder was controlled through a microcontroller which interfaced with the measurement computer. The measurement computer had the user interface where the desired input parameters were entered before the measurement was started.

\section{A. Azimuth Sampling Rate}

To avoid ambiguities, the radar had to be triggered often enough that the Nyquist sampling criterion was satisfied for azimuth sampling. Hence a maximum step size needs to be calculated where a radar measurement (azimuth sample) needs to be taken. This shall now be briefly explained. The received signal after down-conversion can be represented as [5],

$$
s_{\mathrm{IF}}\left(X_{\mathrm{azi}}, K_{\mathrm{r}}\right)=A_{\mathrm{IF}} \cdot \mathrm{e}^{-\mathrm{j} K_{\mathrm{r}} \sqrt{\left(X_{\mathrm{azi}}-X_{\mathrm{t}}\right)^{2}+Y_{\mathrm{t}}^{2}}},
$$

where $A_{\mathrm{IF}}$ represents the amplitude of the signal, $X_{\mathrm{t}}$ and $Y_{\mathrm{t}}$ are the coordinates of the pixel being imaged, $X_{\mathrm{azi}}$ is the azimuth position of the radar along the track and, 


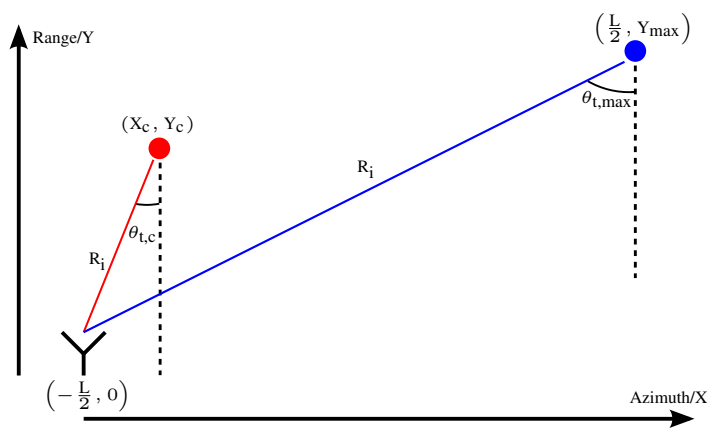

Fig. 1. Illustration of measurement geometry. $\theta_{\mathrm{t}, \max }$ is the maximum aspect angle and $R_{i}$ is the slant range to each target or pixel.

$$
K_{\mathrm{r}}=\frac{4 \pi}{\mathrm{c}_{0}}\left(f_{\text {start }}+\gamma \hat{t}\right)
$$

with $f_{\text {start }}$ being the start frequency of the chirp, $\gamma$ the chirp rate and $\hat{t}$ the fast time. The instantaneous frequency of the received signal from the target at $\left(X_{\mathrm{t}}, Y_{\mathrm{t}}\right)$ can be found by taking the partial derivative with respect to the azimuth domain [9],

$$
\begin{aligned}
k_{\mathrm{x}}\left(X_{\mathrm{azi}}\right) & =\frac{\partial}{\partial X_{\mathrm{azi}}}\left[-\mathrm{j} K_{\mathrm{r}} \sqrt{\left(X_{\mathrm{azi}}-X_{\mathrm{t}}\right)^{2}+Y_{\mathrm{t}}^{2}}\right] \\
& =\frac{K_{\mathrm{r}}\left(X_{\mathrm{t}}-X_{\mathrm{azi}}\right)}{\sqrt{\left(X_{\mathrm{azi}}-X_{\mathrm{t}}\right)^{2}+Y_{\mathrm{t}}^{2}}} \\
k_{\mathrm{x}} & =K_{\mathrm{r}} \sin \left[\theta_{\mathrm{t}}\left(X_{\mathrm{azi}}\right)\right] \\
\text { where } \quad \theta_{\mathrm{t}}\left(X_{\mathrm{azi}}\right) & =\tan ^{-1}\left(\frac{X_{\mathrm{t}}-X_{\mathrm{azi}}}{Y_{\mathrm{t}}}\right)
\end{aligned}
$$

is the aspect angle when the radar is located at $\left(X_{\text {azi }}, 0\right)$ and the target pixel is located at $\left(X_{\mathrm{t}}, Y_{\mathrm{t}}\right)$ [9]. The aspect angle is illustrated in Fig. 1, where the radar is shown at the start of its track at position $\left(-\frac{\mathrm{L}}{2}, 0\right)$ and the blue target $\left(\frac{\mathrm{L}}{2}, Y_{\max }\right)$ is the farthest target, and therefore it has the largest aspect angle.

After applying the Fourier transform in the azimuth domain to Eqn. (1), the received signal becomes [9]

$$
S_{\mathrm{IF}}\left(K_{\mathrm{x}}, K_{\mathrm{r}}\right)=A_{\mathrm{IF}} \cdot \mathrm{e}^{-\mathrm{j} Y_{\mathrm{t}} \sqrt{K_{\mathrm{r}}^{2}-K_{\mathrm{x}}^{2}}-j K_{\mathrm{x}} X_{\mathrm{azi}},}
$$

where $K_{\mathrm{x}}$ denotes the spatial frequency (Doppler) domain with the corner points at $K_{\mathrm{x}} \in\left[k_{\mathrm{x}}\left(-\frac{\mathrm{L}}{2}\right), k_{\mathrm{x}}\left(\frac{\mathrm{L}}{2}\right)\right]$ [9]. The Doppler support band thus becomes [9],

$$
\Omega_{\mathrm{t}}=\left[-K_{\mathrm{r}} \cdot \sin \left[\theta_{\mathrm{t}}\left(\frac{\mathrm{L}}{2}\right)\right], K_{\mathrm{r}} \cdot \sin \left[\theta_{\mathrm{t}}\left(\frac{\mathrm{L}}{2}\right)\right]\right] .
$$

The complete support bandwidth for the entire scene is thus the union of the support bands of each receive signal $\left(S_{\mathrm{IF}}\right)$ [9],

$$
\begin{aligned}
\Omega_{\mathrm{s}} & =\cup \Omega_{\mathrm{t}} \\
& =\cup\left[-K_{\mathrm{r}} \cdot \sin \left[\theta_{\mathrm{t}}\left(\frac{\mathrm{L}}{2}\right)\right], K_{\mathrm{r}} \cdot \sin \left[\theta_{\mathrm{t}}\left(\frac{\mathrm{L}}{2}\right)\right]\right] \\
& =\left[-K_{\mathrm{r}} \cdot \sin \theta_{\mathrm{t}, \max }, K_{\mathrm{r}} \cdot \sin \theta_{\mathrm{t}, \max }\right],
\end{aligned}
$$

where $\theta_{\mathrm{t}, \max }=\tan ^{-1}\left(\frac{\mathrm{L}}{\mathrm{Y}_{\max }}\right)$ is the largest aspect angle as shown in Fig. 1. The Nyquist sample spacing in azimuth is thus [9],

$$
\Delta x \leq \frac{\pi}{K_{\mathrm{r}} \cdot \sin \theta_{\mathrm{t}, \max }} .
$$

For the measurements presented here, the sample spacing, $\Delta x$, was calculated to be $1.5 \mathrm{~mm}$. Therefore it was necessary to ensure that the script (running on the measurement computer) controlling the radar and rotary encoder be time and resource efficient. This was accomplished with multi-threading, with one thread triggering the radar, another fetching the data from the buffer in the radar and the third controlling the micro-controller connected to the rotary encoder. The threads were coordinated through signalling between them. To tie the radar and the rotary encoder together, a hardware trigger from the radar was fed into the micro-controller which was interfacing with the rotary encoder. Hence the micro-controller could maintain a time-line of when each chirp signal was transmitted and save the corresponding trajectory information at that instant.

The measurement computer also maintained a separate timeline of when the instructions to the radar were sent. Hence, by correlating the two time-lines during signal processing, one could double check if the radar and the rotary encoder were actually synchronised and delivering valid, logical results or whether to discard a particular measurement as it was invalid. The difference between the time-lines was found to be consistently within a tolerance of $10 \mu$ s. Hence the portion of the measurement setup responsible for acquisition of measurement data was validated and verified to conform to the stringent requirements for processing of SAR images.

\section{B. Implementation of a Realistic Processing Algorithm}

To create a realistic SAR implementation, the measurements were processed such that they were being acquired in real time. Thus the measurements were done in blocks with a gap in between, which is needed to transfer the data, as shown in Fig. 2. This gap would also be present in a realistic automotive setup, as the radar would not be dedicated for SAR measurements solely, rather it would switch between applications. The blocks are illustrated in Fig. 2, where the blue chirps represent the block of measurements which precede the red chirps in time. Similarly, all of the measurements can thus be broken down into blocks and processed block-wise. The first block (blue chirps) are copied into a matrix, and padded with zeros. In Fig. 2, the blue space represents the signal data from blue chirps and the white space represents zeros. Then a second matrix is created, and the red chirps are copied into it as shown in Fig.2. This process is repeated for all the blocks of chirps until all signal data has been copied into the matrices. In the next step, the missing signal data (shown in Fig. 2 by the dotted ramps) are recovered through compressed sensing using IMAT with a stopping criteria defined in [7]. This improves the dynamic range as well as the clarity of the SAR images as without recovering the missing signals, the data is processed with the gaps and this decreases the amount of target data. This can lead to a reduction of the resolution of the SAR images as well as deteriorating the dynamic range of the image. This is thus avoided in this approach.

Now that the signal data has been sorted, each matrix is individually processed, just like it would be in a realistic real 


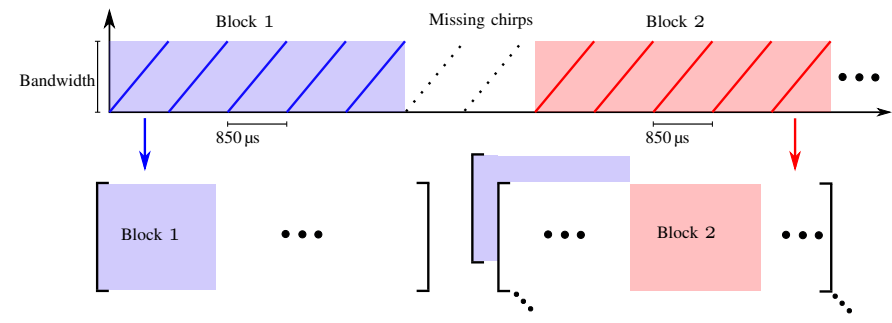

Fig. 2. Realistic implementation of the processing algorithm.

TABLE I

PROCESSING TIMES

\begin{tabular}{|l|l|}
\hline Algorithm & Processing Time \\
\hline \hline Backprojection & $13.0 \mathrm{~s}$ \\
\hline RMA (not optimised) & $2.6 \mathrm{~s}$ \\
\hline Sub-Aperture & $1.9 \mathrm{~s}$ \\
\hline
\end{tabular}

time implementation of automotive SAR. Hence all the motion compensation steps as well as the correction for non-uniform azimuth sampling [9] is done for each matrix before they are input into the range migration algorithm (RMA) [5]. Finally, all the processed matrices are stitched together to produce one complete SAR image of the target scenery. This realistic implementation of the RMA is referred to as the sub-aperture approach since it involves breaking up the trajectory into subapertures and processing each independently. Since each time a smaller matrix is processed, the processing time is reduced as can be seen from Table I. The backprojection algorithm is the slowest, while the difference between RMA without optimisation and the sub-aperture algorithm is smaller.

It is pertinent to mention here that with this demonstrator it is only possible to precisely identify the relative positions of objects, that is the positions of different targets with respect to each other rather than their absolute positions. This is due to the fact that the absolute position, on the trajectory, of a particular radar measurement cannot be determined. Rather, for every radar measurement, only the relative position with respect to the previous measurement is very accurately known. Coupled with the fact that all measurements with the SAR demonstrator are unique and cannot be reproduced exactly, since the demonstrator is moved by a human (as opposed to a precise motor [6]) and it is not possible to reproduce the exact same movements (velocities, accelerations and their durations), leads to the fact that the accuracy of the processed SAR image has to be gauged by the relative distances between points in the target scenery.

\section{Measurement Results}

The target scenery for the measurements are shown in Fig. 3. The SAR demonstrator can be seen in Fig. 3a. The radar sensor is shown placed at right angles to the path direction of the trolley. Also visible in the photo is the target, the rear view of the parked car.

The processed SAR images of the setup from Fig. 3a are shown in Fig. 4. Fig. 4a is the image processed by the implementation described in Section II-B which uses RMA as the image forming algorithm.

The relative positions of the points on the car in the processed image correspond very well with the ground truth measurements of the measurement setup taken before the measurement was carried out. The contour of the car can be made out easily and it can be seen that the flat sides of the rear of the car cause strong specular reflection. It is also possible to see some details on the two sides of the car like the wheel assembly, though it has a much lower amplitude relative to the flat sides of the car.

To compare the results delivered by the sub-aperture algorithm developed, the same measurements were also processed by the back-projection (BP) algorithm [8]. The BP algorithm is often used as a benchmark to gauge the quality of the processed images, since the BP processes images in time domain, hence it does not lose resolution due to quantisation or rounding off.

Comparing the image generated by the BP algorithm and that by the sub-aperture approach, one can see hardly any differences between the contour of the car. Both of the algorithms have delivered images of the target with the identical information. Hence for this perspective of the car, the subaperture approach is capable of delivering images which are as accurate as the BP algorithm.

The processed SAR images of the scenario from Fig. 3b, with the two cars parked next to each other, can be seen in Fig. 5. The gap between the two cars was measured to be $60 \mathrm{~cm}$.

The image obtained from the sub-aperture algorithm is illustrated in Fig. 5a. The gap between the two cars can also be seen to be approximately $60 \mathrm{~cm}$. The details from the side of the car which were visible in Fig. 4 for a single car, are not readily visible here due to them being obstructed from view for most of the length of the aperture. Comparing the image to the result obtained from the BP algorithm (Fig. 5b), there are hardly any differences that can be seen. Therefore it can inferred that the sub-aperture algorithm in conjunction with RMA can deliver high resolution SAR images which are at par with those obtained from time domain (for example here: BP) based processing algorithms.

\section{CONCLUSION}

It has been successfully shown that automotive SAR is a legitimate technique through which higher resolution radar images of the environment around a car can be obtained. In this work, SAR images were obtained using very basic hardware, though the implementation in a car would benefit from the plentiful radar and trajectory acquisition sensors which are already built-in, thus avoiding additional hardware costs. Additionally, it has been shown that automotive SAR can be made faster through smarter processing algorithms like the sub-aperture approach and the missing signals can be recovered with the help of compressed sensing. 


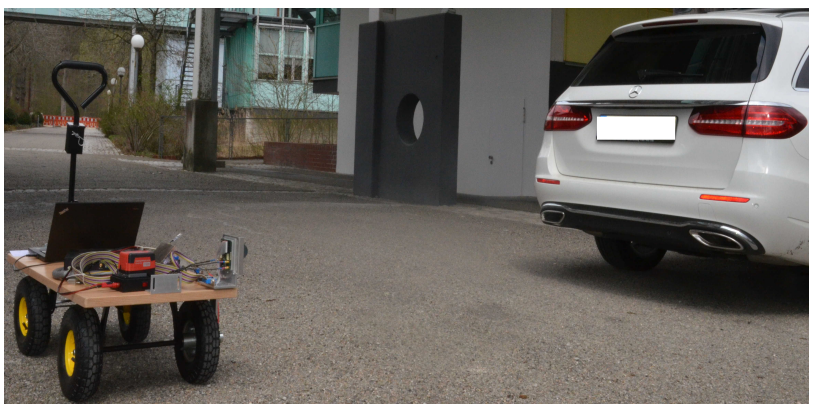

(a) Back view of car with the SAR demonstrator visible as well.

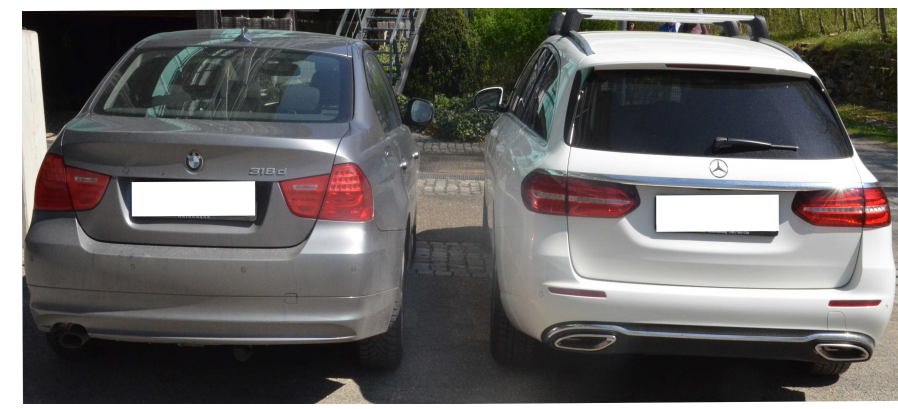

(b) Two cars parked next to each other with a gap of $60 \mathrm{~cm}$. A common parking lot scenario.

Fig. 3. Outdoor measurement scenarios.

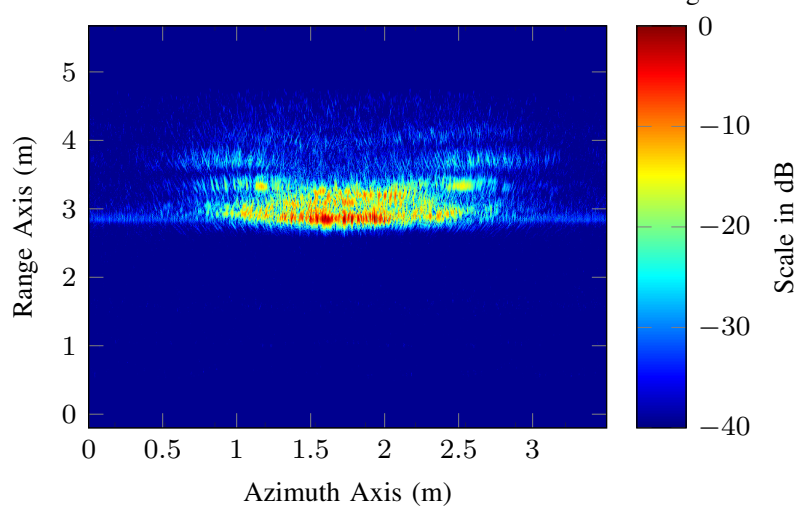

(a) SAR image obtained from the sub-aperture algorithm.

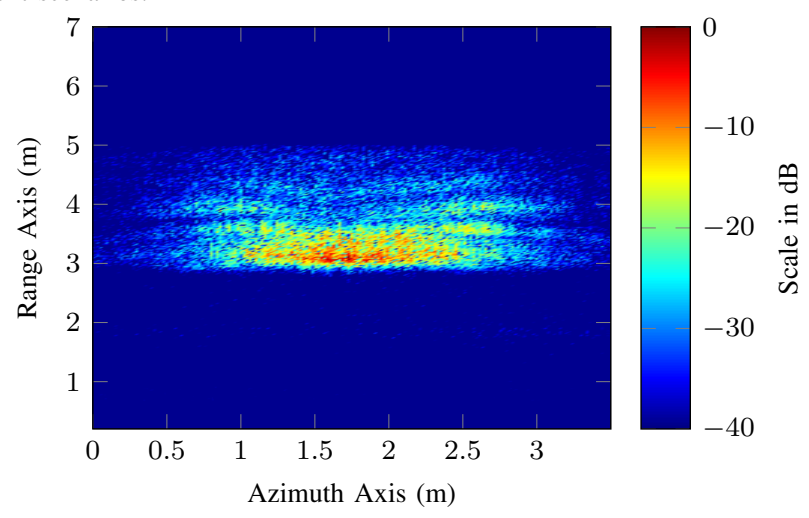

(b) SAR image obtained from the backprojection algorithm.

Fig. 4. SAR images corresponding to measurement scenario from $3 \mathrm{a}$.

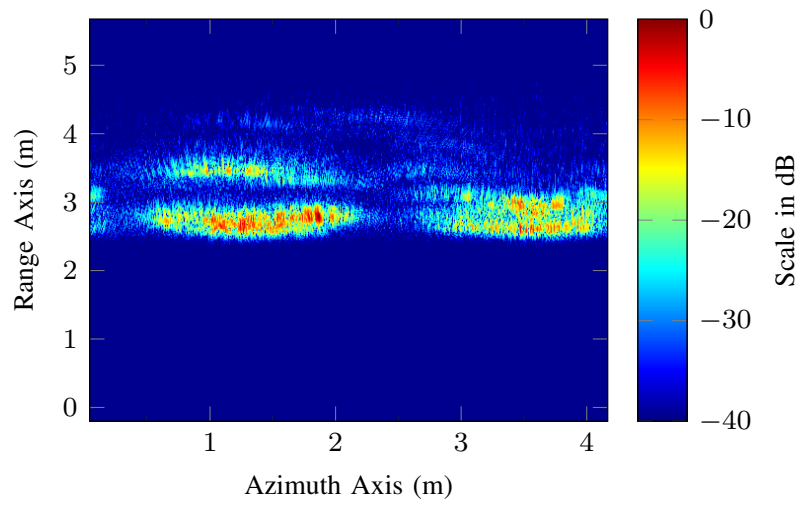

(a) SAR image obtained from the sub-aperture algorithm.

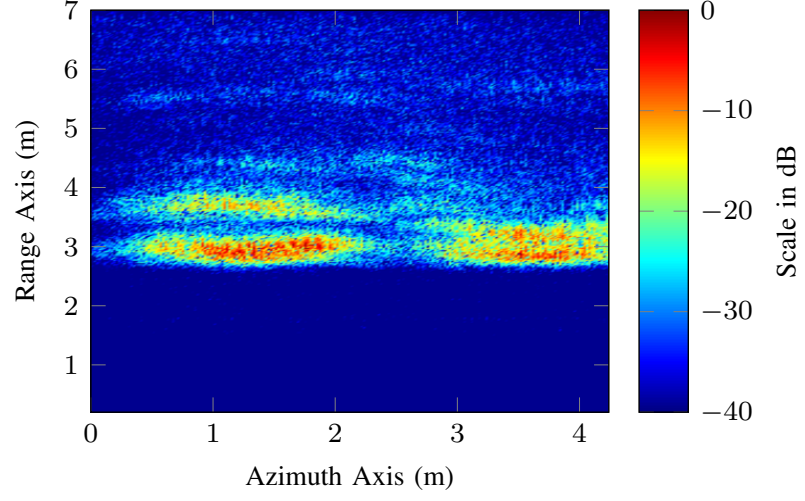

(b) SAR image obtained from the backprojection algorithm.

Fig. 5. SAR images corresponding to measurement scenario from $3 \mathrm{~b}$.

\section{REFERENCES}

[1] W. Huaming et al., "Motion Compensation with one-axis Gyroscope and two-axis Accelerometer for Automotive SAR," in GeMiC, 2011, pp. 1-4.

[2] R. Feger et al., "Experimental Verification of a 77-GHz Synthetic Aperture Radar System for Automotive Applications," in International Conference on Microwaves for Intelligent Mobility (ICMIM), 2017.

[3] A. Laribi et al., "Performance Investigation of Automotive SAR Imaging," in International Conference on Microwaves for Intelligent Mobility (ICMIM), 2018.

[4] F. Harrer et al., "Synthetic Aperture Radar Algorithm for a Global Amplitude Map," in 14th Workshop on Positioning Navigation and Communications (WPNC), Oct. 2017, pp. 1-6.

[5] G. L. Charvat, Small and Short-Range Radar Systems. CRC Press, 2014.

[6] H. Iqbal, B. Sajjad, M. Mueller, and C. Waldschmidt, "SAR Imaging in an Automotive Scenario," in 15th Mediterranean Microwave Symposium (MMS), 2015, pp. 1-4.

[7] F. Roos et al., "Data Rate Reduction for Chirp-Sequence Based Automotive Radars using Compressed Sensing," in 11th GeMiC, 2018

[8] E. C. Zaugg et al., "Generalized Frequency Scaling and Backprojection for LFM-CW SAR Processing," IEEE Transactions on Geoscience and Remote Sensing, 2015.

[9] M. Soumekh, Synthetic Aperture Radar Signal Processing with MATLAB Algorithms. Wiley, 1999. 